\title{
Antibiotics and Drug Pharmacology
}

\section{Stephen Adeniyi ADEFEGHA*}

Department of Biochemistry, Federal University of Technology, Akure, Ondo State, Nigeria

*Corresponding Author: Stephen Adeniyi ADEFEGHA, Department of Biochemistry, Federal University of Technology, Akure, Ondo State, Nigeria.

Received: October 16, 2019; Published: October 21, 2019

DOI: $10.31080 /$ ASPS.2019.03.0424

\section{Introduction}

Drugs are chemicals which when administered to living organisms produce a biological effect. The term antibiotic was derived from the word "antibiosis", which literally means "against life". Antibiotics, in the past, were considered to be organic compounds produced by one microorganism which are toxic to other microorganisms by selectively killing or inhibiting the growth of other microorganisms [1]. In modern terms, this definition includes antimicrobials produced through synthetic means partly (semi-synthetic) or wholly (synthetic) [2].

Some antibiotics are able to kill bacteria completely while some only inhibit their growth. Bactericidal are those that kill bacteria while bacteriostatic are those that inhibit bacterial growth [3]. In September 1928, the first antibiotics (Penicillin) was accidentally discovered from a soil inhabiting fungus Penicillium notatum by late Sir Alexander Fleming, an English Bacteriologist, but its discovery was first reported in 1929 [4], it was purified in 1940 by Florey and Chain, who purified it by freeze drying and a Nobel prize was won in 1945. Clinical trials were first conducted on humans in 1940 and during World War II, penicillin saved $12-15 \%$ of lives $[1,5]$.

Selman Waksman discovered Streptomycin in 1943 which is active against all Gram-negatives and it is the first antibiotic active against Mycobacterium tuberculosis. Most severe infections are caused by gram-negatives and Mycobacterium tuberculosis.

\section{Basic anatomy of bacterial cell}

The cytoplasmic membrane of Gram-positive bacteria is surrounded by a tough and rigid mesh called the cell wall. The cell wall is a tough layer that gives bacterium its shape, it also shield it from both mechanical and from osmotic stresses. But the Gram-negative bacteria have a thin cell wall that is surrounded by another membrane called outer membrane (OM) aside from the cytoplasmic membrane. The $\mathrm{OM}$ is another protective layer which prevents many substances from entering into the bacterium, but it contains some channels called porins. The porins allow the entry of various molecules into the bacterium such as drugs [6]. The periplasm is the space between the outer membrane and cytoplasmic mem- brane. The cytoplasmic membrane prevents ions from flowing into or out of the cell; this maintains the cytoplasmic and bacterial components in a well defined space. Gram staining is based on the ability of bacteria cell wall to retain crystal violet dye during solvent treatment. The cell walls for Gram-positive microorganisms have a higher peptidoglycan and lower lipid content than Gram-negative bacteria [7].

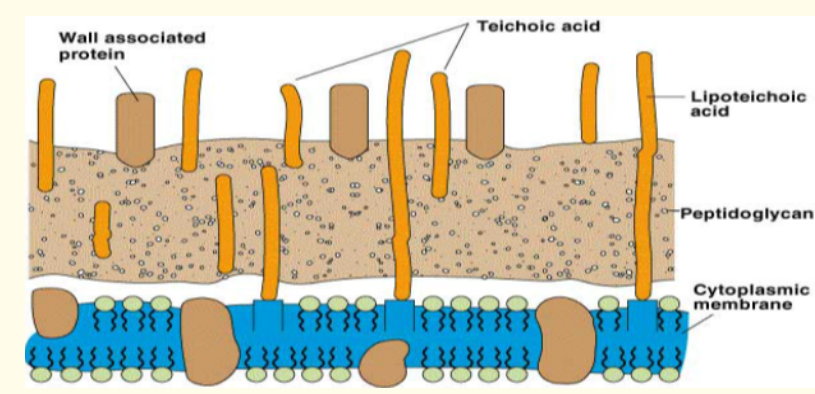

Figure 1: Gram-positive Bacteria Cell.

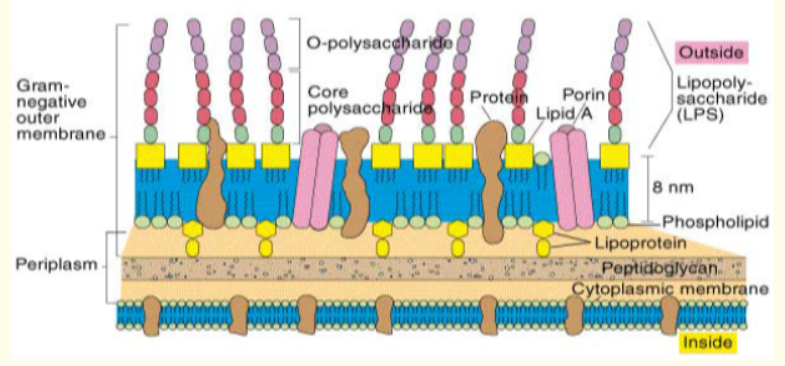

Figure 2: Gram-negative Bacteria Cell [8].

Common Gram-positive organisms are Streptococcus, S. aureus, Bacillus anthracis, Clostridium botulinum. Common Gram-negative organisms are Klebsiella pneumonia, Shigella, Yersinia pestis, Salmonella typhimurium, Salmonella enteriditis. 


\section{Classification of antibiotics}

There are several ways of classifying antibiotics but the most common classification are based on their molecular structures, spectrum of activity and mode of action [8]. The uncommon classification includes: route of administration, which can be injectable, topical and oral). The allergic-potential side effects, toxicity and effectiveness of antibiotics in the same structural class will show the same pattern.

\section{Classification based on molecular structures}

Some common classes of antibiotics based on their molecular or chemical structures include Beta-lactams, Tetracyclines, Macrolides, Quinolones, Sulphonamides, Aminoglycosides, Oxazolidinones and Glycopeptides [9-11].

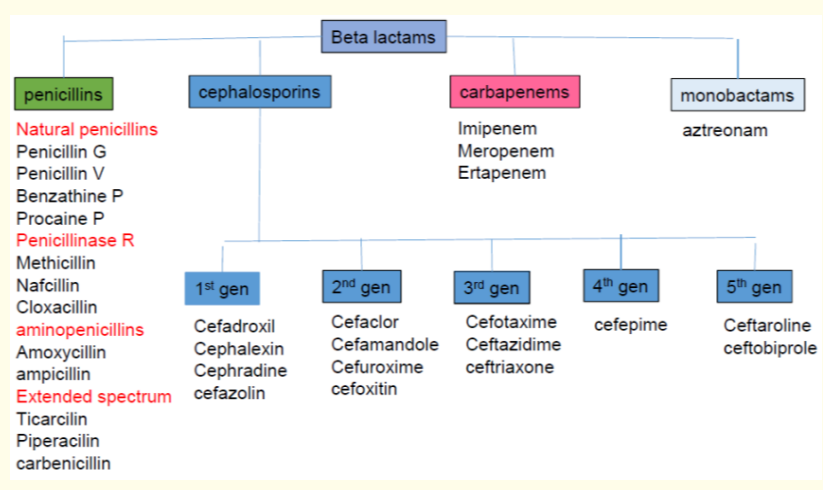

Figure 3: Beta-lactam family [2].

\section{Classification based on mechanisms of action for antibiotics}

There are differences in cell target of antibiotics and these have led to the difference in mechanism of action. The mechanisms of action of some common antibiotics will be discussed under this section.

\section{Antibiotics targeting cell wall}

- Inhibition of peptidoglycan cross-linking (beta-lactams)

- Inhibition of peptidoglycan synthesis (vancomycin).

The peptidoglycan, also called murein in older sources, of the cell wall surrounding the bacterial cells are made of long sugar polymers. Peptidoglycan has some cross-linking peptide bonds known as $\beta-(1-4)-\mathrm{N}$ - acetyl Hexosamine $[13,14]$. The bacteria must necessarily synthesize the peptidoglycan for it to stay alive and they do this by the activity of Penicillin Binding Proteins (transpeptidases and transglycosylase). The glycan strands of existing peptidoglycan molecule are extended by these two enzymes by adding disaccharide pentapeptides and they also cross-link strands of immature peptidoglycan units [15]. The enzyme transglycosidases catalyzes the cross-linking of the glycan strands by of the peptidoglycan and there is an extension of the peptide chains from the polymers sugar and form cross links, that is, one peptide to another [16]. The Dalanyl-alanine portion of peptide chain is cross linked by glycine residues in the presence of penicillin binding proteins (PBPs) [17], this strengthens the cell wall. The Beta-lactams and the glycopeptides inhibit cell wall synthesis and peptidoglycan cross-linking.

\section{Beta-lactam antibiotics}

The $\beta$-lactam majorly targets the Penicillin Binding Proteins. The $\beta$-lactam ring is an analog of the D-alanyl D-alanine portion of peptide chain that binds with the PBPs. The $\beta$-lactam mimics Dalanyl D-alanine and is bounded by PBP. Therefore, the PBPs are not available for the synthesis of new peptidoglycan and this disruption leads to the lyses of bacterium cell wall and ultimately the death of the bacteria [18]. Drugs like penicillin (examples are penicillin and amoxicillin), cephalosporin (examples are cephalexin and cefdinir) and carbapenem are able to block the cross-linking of peptidoglycan units by inhibiting the peptide bond formation catalyzed by the penicillin Binding Proteins [19]. Examples of Betalactam antibiotics with increased activity are amoxicillin/clavulanate, ceftazidime/avibactam.

\section{Glycopeptides}

The glycopeptides binds to D-alanyl D-alanine portion of peptide side chain of the precursor peptidoglycan subunit. The drug molecule vancomycin prevents binding of this D-alanyl subunit with the Penicillin Binding Proteins, and hence inhibits cell wall synthesis $[18,20]$. Most of the antibiotics that belong to the glycopeptide class of antibiotics (e.g. vancomycin) inhibit the synthesis of peptidoglycan which leads to bacterial growth inhibition. This is done by binding themselves to peptidoglycan units, as well as blocking the penicillin binding proteins (transglycosylase and transpeptidase) activities [16].

Disruption of cell membrane

\section{Polymyxins}

These classes of antibiotics which damages cell membranes of bacteria are specific in each microbial group because of the differences in the types of lipids in their cell membranes. Daptomycin, for example, depolarizes calcium-dependent membrane, which leads to the ceasing of the synthesis of macromolecules and disruption of the cellular membrane in bacteria [21]. The effective binding of polymyxins to the lipid moiety of the lipopolysaccharide in the bacterial cell causes disintegration of bacterial cell membrane [22]. The disintegration of the cell membrane is used against Gramnegatives for local treatment and it has a narrow spectrum.

\section{Inhibition of protein synthesis}

- At 30 S ribosomal subunit (aminoglycosides, tetracyclines)

- At 50 S ribosomal subunit (macrolides, chloramphenicol).

The central dogma of molecular biology is the transcription of DNA into RNA and the translation of RNA into protein. First, the genetic information in the bacterial DNA is transcribed into an RNA 
molecule called messenger RNA (mRNA) through transcription. Then, ribosome synthesizes proteins present in mRNA through translation. The biosynthesis of Protein is catalyzed by cytoplasmic factors and ribosomes. The $30 \mathrm{~S}$ and $50 \mathrm{~S}$ ribonucleoprotein subunits make up the bacterial 70S ribosome [23]. Either of these two ribonucleoprotein subunits is the target of the antimicrobials that inhibit protein biosynthesis $[24,25]$. Some of these antimicrobials are discussed below;

\section{Inhibitors of $30 \mathrm{~S}$ subunit}

- Aminoglycosides: The aminoglycosides (AGs) gain access into the bacterium by forming pores on the outer membrane of the bacterium. Recall, like charges attract while unlike charges repel, the AGs are positively charged and attached to the outer membrane which is negatively charged. This leads to the formation of the large pores and ultimately allows the antibiotic to penetrate inside the bacterium. For the antibiotics to reach its target, which is the bacterial ribosome, it needs to pass through cytoplasmic membrane which requires energy dependent active bacterial transport mechanism, this requires oxygen and an active proton motive force (PMF). Because of these requirements, AGs work under aerobic conditions and have poor activity against anaerobic bacteria. These antibiotics have synergism with the antibiotics that inhibit cell wall synthesis (e.g. $\beta$-lactam and glycopeptides) because this allows greater penetration of AGs within the cell and at low dosages. AG's interact through hydrogen bonds with the $16 \mathrm{~S}$ ribosomal RNA (rRNA) of the $30 \mathrm{~S}$ subunit near the A site. This leads to the misreading and premature termination of translation of mRNA [7].

- Tetracyclines: Tetracyclines (such as tetracycline, doxycycline, chlortetracycline or minocycline) act upon the conserved sequences of the $16 \mathrm{~S}$ rRNA of the $30 \mathrm{~S}$ ribosomal subunit and prevent the binding of transfer RNA (tRNA) to the A site of the ribosome $[23,26]$.

\section{Inhibitors of 50S subunit}

- Chloramphenicol: It acts upon the conserved sequences of the peptidyl transferase cavity of the $23 \mathrm{~S}$ ribosomal RNA (rRNA) of the 50S subunit. Hence, it inhibits the protein synthesis by preventing binding of transfer RNA (tRNA) to the A site of the ribosome $[23,24]$.

- Macrolides: They target the conserved sequences of the peptidyl transferase center of the 23S rRNA of the 50S ribosomal subunit at translocation (early stage of protein synthesis). This action results in the premature detachment of the incomplete peptide chains. Lincosamides, streptogramins B and Macrolides show similar mechanism of action [7]. Examples of macrolides are azithromycin and erythromycin.

- Oxazolidinones: Oxazolidinones affect protein synthesis at several stages; they suppress $70 \mathrm{~S}$ inhibition and interact with peptidyl-t-RNA and also inhibit protein synthesis by binding to $23 \mathrm{~S}$ rRNA of the 50S subunit. Linezolid is a recently approved member of novel class of antibiotic of this group which is completely synthetic $[27,28]$.

\section{Inhibition of nucleic acid}

- Inhibition of folic acid synthesis (sulphonamides, trimethoprim)

- Inhibition of DNA gyrase (fluoroquinolones)

- Inhibition of RNA synthesis (rifampin).

\section{Folic acid metabolism inhibitors}

Some antibiotics (e.g. sulphonamides and trimethoprim) mimic a substrate needed for cellular metabolism of bacteria, that is, they are analog of this substrate. This makes bacterial enzymes to react or attach themselves to the antibiotic instead of the normal substrate. Tetrahydrofolate is required for the synthesis of folic acid in bacterial cells; sulphonamides mimic this substrate (Tetrahydrofolate) [29]. Folic acid is vital in the metabolism of nucleic acid and amino acids; for this reason, sulphonamides ultimately disrupt the production of nucleic acids (DNA and RNA) and amino acids [29]. Each of these drugs inhibits distinct steps in folic acid metabolism. There is a reduced mutation rate for resistance against these drugs by the bacterium when sulpha drugs are combined with trimethoprim, because each of the drugs act on a distinct step of the biosynthetic pathway of folic acid [23]. Sulfonamide has higher affinity for the enzyme, dihydropteroate synthase than p-amino benzoic acid which is the natural substrate, so it inhibits in a competitive manner. Trimethoprim inhibits the enzyme dihydrofolate reductase at a later stage of folic acid synthesis [23].

\section{Quinilones}

Bacterial DNA gyrase is inhibited by fluoroquinolones (FQ). Bacterial DNA gyrase is an enzyme that nicks the double stranded DNA, then introduces negative supercoils and reseals the nicked ends. The DNA gyrase has two A subunits and two B subunits. The nicking of DNA is done by the A subunit, the negative supercoils are introduced by $B$ subunit and the nicked ends are resealed by the A subunit. This process prevents the excessive positive supercoiling of the strands when they separate so that transcription or replication can occur. The A subunit is binded with high affinity by FQ which interferes with the subunit's strand cutting and resealing function. But the main target of FQ in Gram-positive bacteria is topoisomerase IV. The topoisomerase IV nicks and separates daughter DNA strand after DNA replication. Greater affinity for this enzyme by the FQ may confer higher potency against Grampositive bacteria. Instead of DNA gyrase or topoisomerase IV in the bacterial cell, mammalian cells possess topoisomerase II, which has very low affinity for FQ, this leads to low toxicity to mammalian cells $[23,26]$. 


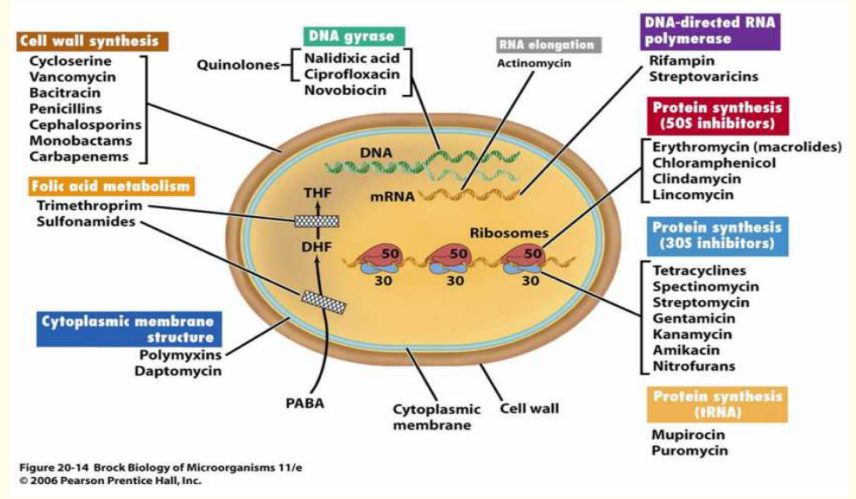

Figure 4: Antibiotic target sites [8].

\section{Pharmacokinetics of antibiotics}

Pharmacokinetics (PK) refers to the study of concentration changes of a drug over a period of time. After the administration of a drug, drugs undergoes ADME (absorption, distribution, metabolism and excretion) processes that condition the kinetics of the drug and the concentration-time profile, which may be characterized by the PK parameters, such as total body clearance, volume of distribution, protein binding or bioavailability [30,31].

\section{Aminoglycosides}

Aminoglycosides route of administered is majorly via parenteral except cases of intestinal infections or indication for decontamination. aminoglycosides upon absorption into the bloodstream, they have low volume of distribution $(<0.3 \mathrm{~L} / \mathrm{kg}$ ) thus aminoglycosides are mainly distributed in blood plasma. In the blood, aminoglycosides bind weakly to plasma protein (about only 30\%) and possess a plasma elimination half-life of approximately two hours. They are excreted extensively via kidney (renal excretion) in urine in the unchanged biological active form (only $<10 \%$ is biotransformed). But small fraction was found to be eliminated through bile $0.5-2 \%$ of the administered dose). The PK of elimination is independent of the dose and route of administration. Aminoglycosides are distributed poorly into tissues due to poor diffusion, but they are well distributed into peritoneal, pleural and pericardial fluid and synovial effusions due to good diffusion. In the above aforementioned fliuds, aminoglycosides attain a concentration of 25 to $50 \%$ of levels in serum; renal accumulation occurs particularly in the cortex [32].

\section{Tetracycline}

Tetracyclines are administered via oral route, while doxycicline and minocycline can be given either orally or intravenously. Tetracycline drugs are distributed in tissues based on their lipophilicity. In the blood stream, they bind moderately to plasma protein in the range of about $20 \%$ to $60 \%$. Tetracyclines administered via oral route are absorbed in the stomach and proximal small intestine. Oral bioavailability of these compounds is relatively high, ranging from $75 \%$ to $100 \%$. Food reduces the absorption of both tetracycline and doxycycline up to $50 \%$. They have plasma elimination Half-life of 8-25 hours. They are eliminated by both hepatic and renal mechanisms, they are filtered by the gomerulus but due to their high lipophilicity, they are reabsorbed. Tetracycline class are not effectively dialyzed, thus dose adjustment is not necessary in patients with renal impairment [33].

\section{Macrolide}

Macrolides are administered via oral route. After oral administration, they are readily absorbed from the gastrointestinal tract if not inactivated by gastric acid. Macrolide have high bioavailability. They also possess high volume of distribution (1-2.5 L/kg) which reflects their extensive tissue penetration. In the bloodstream, 75\% of the administered dose is bounded to plasma Proteins. They actually accumulate within many cells, including macrophages, in which they may be $\geq 20$ times the plasma concentration. Macrolides tend to concentrate in the spleen, liver, kidneys, and particularly the lungs. They enter pleural and peritoneal fluids but not the cerebrospinal fluid (only $2-13 \%$ of plasma concentration unless the meninges are inflamed). They concentrate in the bile and milk. Macrolide are inactivated by metabolic activities of the liver but the relative proportion of the drug inactivated by metabolism depends on the route of administration and the particular antibiotic. For example, after oral administration of erythromycin, about $80 \%$ of the administered dose undergoes metabolic inactivation, whereas tylosin appears to be eliminated in an active form. Macrolide antibiotics and their metabolites are excreted mainly in bile $(>60 \%)$ and often undergo enterohepatic cycling. Urinary clearance may be slow and variable (often $<10 \%$ ) but may represent a more significant route of elimination after parenteral administration [34].

\section{$\beta$-lactams Antibiotics}

- Penicillin's: Penicillin's are administered mainly via oral route. After oral administration, penicillin's are absorbed in the stomach and proximal small intestine. In the bloodstream, penicilins have different values of protein binding; with the exception of piperacillin and clavulanic acid that are not orally absorbed, the gastrointestinal absorption is $>50 \%$ for almost all drugs considered. They are minimally metabolized and the main route of elimination is renal excretion as parent drug [35].

- Cephalosporins: Cephalosporins after administration, while in the bloodstream, bind to plasma proteins in different degrees. The reported value for protein binding range from $6 \%$ for cephradine to $92 \%$ for cefazolin. Cephalosporins generally distribute well into the lung, kidney, urine, synovial, pleural, and pericardial fluids. Penetration into the CSF of some third generation cephalosporins (cefotaxime, ceftriaxone, and ceftazidime) is adequate to effectively treat bacterial meningitis. Cephalosporins are extensively elimi- 
nated from the body via the kidney, though a few exceptions include cefoperazone and ceftriaxone which have significant biliary elimination. Biliary excretion of cephalosporins is highly dependent on molecular weight in rats: less than $15 \%$ of the dose is excreted into the bile for cephalosporins with a molecular weight of less than 450, but those with a molecular weight of more than 450 exhibit 15 to $100 \%$ recovery in bile. In addition, their elimination pathway is mainly excretion into bile and/or urine with minimal metabolism in the body [35].

\section{Resistance to antibiotics}

Antimicrobials have helped us deal with disease, but on the other hand, the improper use of these antimicrobials has created new difficulties. Antibiotic resistance is the ability of bacteria to protect themselves against the effects of an antibiotic. The appropriate and inappropriate use of antibiotics the past years has lead to increased occurrence and spread of bacteria that are resistant to antibiotics. Resistance against antibiotics can either be natural or acquired.

The under listed human factors has contributed to the development of resistance against antibiotics by bacteria; the prescribing antibiotics too often, too long therapy or too low dose, the stop of medication before completing the therapy, usage of antibiotics in animal husbandry and the spread of resistant hospital strains due to unhygienic lifestyle. The following factors can be considered in limiting resistance; constant exposure to high levels of antibiotic, use of multiple antibiotics and restricted use of antibiotics [36-50].

The following are the common mechanism of antibiotic resistance;

1. Alteration of Targets, this usually affects ribosomes.

2. Alteration of Membrane Permeability, this is caused by the change in the receptor that binds the drug.

3. Development of Enzymes that inactivate antibiotics such as beta-lactamase, aminoglycoside- modifying enzymes and chloramphenicol acetyltransferases. The first $\beta$-lactamase was identified in 1940 [7].

4. Efflux pumps; Membrane proteins of Gram negatives that pump out drug

5. Alteration of Metabolic Pathway; Development of alternate metabolic pathway(s).

Below is a table showing the summary of the resistance mechanism of different antibiotics.

\begin{tabular}{|c|c|c|c|}
\hline Antibiotic class & Resistance type & Resistance mechanism & Common example \\
\hline \multirow[t]{2}{*}{ Aminoglycoside } & Decreased uptake & Changes in outer membrane & $P$ aeruginosa \\
\hline & $\begin{array}{l}\text { Enzymatic } \\
\text { modification }\end{array}$ & AGE's & Gram-negative bacteria \\
\hline \multirow[t]{2}{*}{ Beta-lactams } & Altered PBP & PBP 2a & Mec A in S. aureus, CONS, S. pneumoniae \\
\hline & $\begin{array}{l}\text { Enzymatic } \\
\text { degradation }\end{array}$ & $\begin{array}{l}\text { Penicillinase which are classified as per ambler } \\
\text { classification }\end{array}$ & Gram-negative bacteria \\
\hline Glycopeptides & Altered target & $\begin{array}{l}\text { D-alanyl-alanine is changed to } \\
\text { D-alanyl-D-lactate }\end{array}$ & $\begin{array}{l}\text { Vancomycin resistance in } E \text {. faecium and } \\
\text { E. faecalis }\end{array}$ \\
\hline \multirow[t]{2}{*}{ Macrolides } & Altered target & $\begin{array}{l}\text { Methylation of ribosomal active site with } \\
\text { reduced binding }\end{array}$ & $\begin{array}{l}\text { erm-encoded methylases in S. aureetls, } \\
\text { S. pneumoniae, and S. pyogenes }\end{array}$ \\
\hline & Efflux pumps & Mef type pump & S. pneumoniae and $S$. pyogenes \\
\hline Oxazolidinones & Altered target & $\begin{array}{l}\text { Mutation leading to reduced binding to active } \\
\text { site }\end{array}$ & E. faecium and S. aureus \\
\hline \multirow[t]{2}{*}{ Quinolones } & Altered target & $\begin{array}{l}\text { Mutation leading to reduced binding to active } \\
\text { site(s) }\end{array}$ & $\begin{array}{l}\text { Mutations in gyr A in enteric } \\
\text { Gram-negative bacteria and S. aureus }\end{array}$ \\
\hline & Efflux & Membrane transporters & $\begin{array}{l}\text { Mutations in gyr } \mathrm{A} \text { and par } \mathrm{C} \text { in } \\
\text { S. pneumoniae. Nor-A in } S \text {. atreus }\end{array}$ \\
\hline \multirow[t]{2}{*}{ Tetracyclines } & Efflux & New membrane transporters & $\begin{array}{l}\text { tet genes encoding efflux proteins in } \\
\text { Gram-positive and Gram-negative bacteria }\end{array}$ \\
\hline & Altered target & $\begin{array}{l}\text { Production of proteins that bind to the ribosome } \\
\text { and alter the conformation of the active site }\end{array}$ & $\begin{array}{l}\text { tet }(\mathrm{M}) \text { and tet }(0) \text { in Gram-positive and } \\
\text { Gram-negative bacteria species }\end{array}$ \\
\hline \multirow[t]{2}{*}{ Chloramphenicol } & $\begin{array}{l}\text { Antibiotic } \\
\text { inactivation }\end{array}$ & Chloramphenicol acetyl transferase & CAT in S. pneumonia \\
\hline & Efflux pump & New membrane transporters & $\mathrm{cml} \mathrm{A}$ gene and flo gene efflux in $E$. colt \\
\hline Sulfa drugs & Altered target & Mutation of genes encoding DHPS & E. coli, S. aurens, S. pneumoniae \\
\hline
\end{tabular}

Table: Resistance mechanism of individual antibiotics (Garima et. al., 2017). 


\section{Bibliography}

1. Russell AD. "Types of antibiotics and synthetic antimicrobial agents". In: Denyer S. P., Hodges N. A and German S. P. (eds.) Hugo and Russells pharmaceutical microbiology. 7th Ed. Blackwell Science UK (2004): 152-186.

2. Ebimieowei Etebu and Ibemologi Arikekpar. "Antibiotics: Classification and mechanisms of action with emphasis on molecular perspectives". IJAMBR 4 (2016): 90-101.

3. Walsh C. "Antibiotics: actions, origins, resistance". 1st Ed. ASM Press, Washington, DC (2003): 345.

4. Aminov RI. "A brief history of the antibiotic era: Lessons learned and challenges for the future". Frontier in Microbiology 1 (2010): 1-7.

5. Schlegel HG. "General microbiology". 7th Ed. Cambridge University Press, Cambridge (2003).

6. Hauser AR. Editor: Cell envelope. Antibiotic Basic for Clinicians. 2nd ed. New Delhi: Wolters Kluwer (India) Pvt. Ltd (2015): 3-5.

7. Garima Kapoor., et al. "Action and resistance mechanisms of antibiotics: A guide for clinicians". Journal of Anaesthesiology Clinical Pharmacology 33 (2017): 300-305.

8. Madigan MT and Martinko JM. "Brock biology of microorganisms”. 11th edition. Pearson Prentice Hall Inc (2006).

9. Calderon CB and Sabundayo BP. "Antimicrobial classifications: Drugs for bugs". In: Schwalbe R, Steele-Moore L and Goodwin AC (eds). Antimicrobial susceptibility testing protocols. CRC Press, Taylor and Frances group (2007).

10. van Hoek AHAM., et al. "Acquired antibiotic resistance genes: An overview". Frontier Microbiology 2 (2011): 203.

11. Frank $U$ and Tacconelli E. "The Daschner Guide to In-Hospital Antibiotic Therapy". European standards (2012).

12. Adzitey F. "Antibiotic classes and antibiotic susceptibility of bacterial isolates from selected poultry; a mini review". World's Veterinary Journal 5 (2015): 36-41.

13. Bugg TDH and Walsh CT. "Intracellular steps of bacterial cell wall peptidoglycan biosynthesis: Enzymology, antibiotics, and antibiotic resistance". Natural Product Reports 9 (1992): 199215.

14. Holtje JV. "Growth of the stress bearing and shape maintaining murein sacculus of Escherichia coli". Microbiology and Molecular Biology Reviews 62 (1998): 181-189.
15. Park JT and Uehara T. "How bacteria consume their own exoskeleton (turnover and recycling of cell wall-peptidoglycan)". Microbiology and Molecular Biology Reviews 72 (2008): 211227.

16. Kahne D., et al. "Glycopeptide and lipoglycopeptide antibiotics". Chemical Reviews 105 (2005): 425-448.

17. Reynolds PE. "Structure, biochemistry and mechanism of action of glycopeptide antibiotics". European Journal of Clinical Nutrition 8 (1989): 943-950.

18. Džidic S., et al. "Antibiotic resistance mechanisms in bacteria: Biochemical and genetic aspects". Food Technology and Biotechnology 46 (2008): 11-21.

19. Josephine HR., et al. "The Perfect Pencillin? Inhibition of a bacterial DD-peptidase by peptidoglycan-mimetic beta-lactams". Journal of the American Chemical Society 126 (2004): 8122281223.

20. Grundmann H., et al. "Emergence and resurgence of methicillin resistant Staphylococcus aureus as a public-health threat". Lancet 368 (2006): 874-85.

21. Alborn W., et al. "Deptomycin disrupts membrane potential in growing Staphylococcus aureus". Antimicrobial Agents and Chemotherapy 31 (1991): 1093-1099.

22. Falagas ME., et al. "Resistance to polymyxins: Mechanisms, frequency and treatment options". Drug Resistance Update 13 (2010): 132-138.

23. Yoneyama $\mathrm{H}$ and Katsumata R. "Antibiotic resistance in bacteria and its future for novel antibiotic development". Bioscience, Biotechnology, and Biochemistry 70 (2006): 1060-1075.

24. Vannuffel P and Cocito C. "Mechanism of action of streptogramins and macrolides". Drugs 51 (1996): 20-30.

25. Johnston NJ., et al. "Streptogramin antibiotics: Mode of action and resistance". Current Drug Targets 3 (2002): 335-344.

26. Wise R. "A review of the mechanisms of action and resistance of antimicrobial agents". Canadian Respiratory Journal 6 (1999): 20A-22A.

27. Bozdogan B and Appelbaum PC. "Oxazolidinones: Activity, mode of action, and mechanism of resistance". International Journal of Antimicrobial Agents 23 (2004): 113-119.

28. Lambert PA. "Bacterial resistance to antibiotics: Modified target sites". Advanced Drug Delivery Reviews 57 (2005): 14711485. 
29. Talaro K. P and Chess B. "Foundations in microbiology". 8th Ed. McGraw Hill, New York (2008).

30. Eduardo Asín-Prieto., et al. "Applications of the pharmacokinetic/pharmacodynamic (PK/PD) analysis of antimicrobial agents". Journal of Infection and Chemotherapy 21 (2015): 319-329.

31. Snehal Shah., et al. "Pharmacokinetic considerations and dosing strategies of antibiotics in the critically ill patient". Journal of the Intensive Care Society 16 (2015): 147-153.

32. Briskier A and Veyssier P. "Aminocyclitol and aminoglycoside". In: Press A., editor. Antimicrobial agents; antibacterials and antifungals (2005): 453-457.

33. Agwuh KN and MacGowan A. "Pharmacokinetics and pharmacodynamics of the tetracyclines including glycylcyclines". Journal of Antimicrobial Chemotherapy 58 (2006): 256-265.

34. Nightingale C.H., et al. "Macrolide, Azalide, and Ketolide Pharmacodynamics". In: Ag M.D., editor. Antimicrobial Pharmacodynamics in Theory and Clinical Practice (2002): 205-220.

35. Lorena Baietto., et al. "A 30-years Review on Pharmacokinetics of Antibiotics: Is the Right Time for Pharmacogenetics?". Current Drug Metabolism 15 (2014): 581-598.

36. Bonner DP and Sykes RB. "Structure activity relationships among the monobactams". Journal of Antimicrobial Chemotherapy 14 (1984): 313-327.

37. Brink AJ., et al. "Appropriate use of the carbapenems". SAMJ 94 (2004): 857-861.

38. Brown AG., et al. "Naturally-occurring beta-lactamase inhibitors with antibacterial activity". The Journal of Antibiotics 29 (1976): 668-669.

39. Butterworth D., et al. "Olivanic acids, a family of beta-lactam antibiotics with beta-lactamase inhibitory properties produced by Streptomyces species. Detection, properties and fermentation studies". The Journal of Antibiotics 32 (1979): 287-294.

40. Cassidy PJ., et al. "Epithienamycins. II. Isolation and structure assignment”. The Journal of Antibiotics 34 (1981): 637-648.

41. Heesemann J. "Mechanisms of resistance to beta-lactam antibiotics". Infection 21 (1993): S4-9.

42. Kobayashi F., et al. "Antimicrobial and Beta-lactamase inhibitory activities of carpetimycins A and B, new carbapenem antibiotics". Antimicrobial Agents and Chemotherapy 21 (1982): 536-544
43. Livermore DM., et al. "What remains against carbapenemresistant Enterobacteriaceae? Evaluation of chloramphenicol, ciprofloxacin, colistin, fosfomycin, minocycline, nitrofurantoin, temocillin and tigecycline". International Journal of Antimicrobial Agents 37 (2011): 415-419.

44. Papp-Wallace K., et al. "Carbapenems: past, present, and future". Antimicrobial Agents and Chemotherapy 55 (2011): 4943-4960.

45. Patel G and Bonomo RA. "Status report on carbapenemases: challenges and prospects". Expert Review of Anti-infective Therapy 9 (2011): 555-570.

46. Pegler S and Healy B. "In patients allergic to penicillin, consider second and third generation cephalosporins for life threatening infections". BMJ 335 (2007): 991.

47. Reading $\mathrm{C}$ and Farmer T. "The inhibition of periplasmic $\beta$-lactamase in Escherichia coli by clavulanic acid and other -lactamase inhibitors". McGraw-Hill, New York (1984).

48. Sykes RB and Bonner DP. "Discovery and development of the monobactams”. Clinical Infection Disease 7 (1985): S579-S593.

49. Sykes RB., et al. "Monocyclic $\beta$-lactam antibiotics produced by bacteria”. Nature 291 (1981): 489-491.

50. Torres JA., et al. "Current concepts in antibiotic-resistant gramnegative bacteria". Expert Review of Anti-infective Therapy 5 (2007): 833-843.

\section{Volume 3 Issue 11 November 2019 (C) All rights are reserved by Stephen Adeniyi ADEFEGHA.}

